\title{
Evolución y factores de pronóstico en cáncer diferenciado de tiroides*
}

\author{
Drs. OSVALDO IRIBARREN B. ${ }^{1,2}$, NICOLÁS VELASCO M. ${ }^{1,2}$, PATRICIO HUIDOBRO M. ${ }^{2,4}$, \\ HERNÁN NÚÑ̃Z C. ${ }^{4}$, MAURO VILLALÓN L. ${ }^{4}$, JUAN MADARIAGA G. ${ }^{2,5}$, \\ CARLOS BARRÍA M. ${ }^{5}$, Al. MAGALY SEPÚLVEDA T. ${ }^{3}$
}

\author{
Servicio de Cirugía, Hospital Clínico San Pablo Coquimbo. \\ Escuela de Medicina, Universidad Católica del Norte, Coquimbo. \\ Alumna Escuela de Medicina, Universidad Católica del Norte. \\ Unidad de Oncología, Hospital San Juan de Dios, La Serena. \\ Servicio de Anatomía Patológica, Hospital Clínico San Pablo Coquimbo, Chile.
}

\begin{abstract}
Evolution and prognosis of differentiated thyroid carcinoma

Background: Differentiated thyroid carcinoma has a good prognosis with a $90 \%$ survival at 10 years. Follicular carcinoma is more aggressive than papillary carcinoma Aim: To study the clinical presentation, evolution and prognosis of differentiated thyroid carcinoma. Material and Methods: The pathological registries of differentiated thyroid cancer processed in a pathology service of a general hospital, between 1984 and 2007, were searched. The clinical records of patients were reviewed. Results: One hundred six pathology registries were identified but only 89 patients had complete medical records. Seventy six patients aged $44 \pm 16$ years had a papillary carcinoma and 13 patients aged $42 \pm 15$ years had a follicular carcinoma. After a mean follow up of 53 months, 65 patients with papillary and 12 patients with follicular thyroid cancer were alive. Five patients with papillary carcinoma and one with follicular carcinoma had a local or distant relapse. Calculated actuarial survival at 10 years was 84 and $100 \%$ for papillary and follicular carcinoma, respectively. Ten year survival for stage I, II, III and IV tumors was 100, 92, 68 and 0\%, respectively. Mortality was associated with the presence of distant metastases, a stage IV tumor, vascular or lymphatic tumor infiltration, tumor size, lymph node involvement, extra capsular invasion and a multi focal lesions. The relative risk for mortality increases between 2.7 and 9 times with the presence of lymph node infiltration and distant metastases. Conclusions: The prognosis of differentiated thyroid cancer is related to lymph node infiltration and the presence of distant metastases.
\end{abstract}

Key words: Thyroid cancer, papillary carcinoma, follicular carcinoma, survival.

\section{Resumen}

Los objetivos del presente estudio son identificar la forma de presentación clínica y evolución del cáncer diferenciado de tiroides (CDT); definir la curva de sobrevivencia para ambos tipos histológicos e identificar los factores clínicos e histológicos de mal pronóstico. Se presentan 89 pacientes portadores de

\footnotetext{
*Recibido el 11 de agosto de 2008 y aceptado para publicación el 20 de Octubre de 2008.
}

Correspondencia: Dr. Osvaldo Iribarren B.

Gabriela Mistral 2381, La Serena, Chile.

E-mail: oiribarren@123.cl 
cáncer diferenciado (papilar y folicular) de tiroides (75 CP Y 13 CF) tratados en los Hospitales de Coquimbo y La Serena, entre 1984 y 2007 . La forma de presentación de ambos tumores fue similar. La mediana de seguimiento fue 53 meses. El 75\% de los pacientes tuvo un seguimiento mínimo de 7 años y el 86,4\% de los pacientes (64 cáncer papilar y 12 cáncer folicular) se encuentran vivos y libres de enfermedad; el intervalo libre de enfermedad fue de 52,7 meses con rango entre 3 meses y 16 años. La sobrevida actuarial a 10 años para cáncer papilar es $84 \%$ y, para cáncer folicular $100 \%$. Por estadios, a los 10 años la sobrevida es de $100 \%$ en estadio I, $92 \%$ en estadio II, $68 \%$ en estadio III y $0 \%$ en estadio IV. El riesgo de muerte se asoció a la presencia de metástasis distantes, T4 e infiltración histológica vascular y/o linfática, $\mathrm{p}=0,02$. Otros factores no mostraron asociación: tamaño del tumor $<\mathrm{T} 4$, compromiso de linfonodos, invasión extracapsular y multifocalidad histológica. Sin embargo, el RR se incrementa 2,7 y 9 veces con invasión linfática o con presencia de metástasis, 1,6 veces con el aumento del tamaño tumoral, y 1,33 con multifocalidad neoplásica en la glándula. La presencia de metástasis linfáticas en cáncer papilar representa un RR de 1,22. Aunque el seguimiento de esta casuística es reducido (mediana 5 años), los resultados sugieren que el mal pronóstico se asocia fundamentalmente a compromiso linfático y metástasis a distancia y, por otra parte, a que el planteamiento terapéutico ha resultado efectivo para el control de la enfermedad en estadio I y II.

Palabras clave: Cáncer de tiroides, carcinoma tiroídeo, cáncer.

\section{Introducción}

El cáncer de tiroides es la neoplasia maligna más frecuente del sistema endocrino; sin embargo, su frecuencia de aparición varía entre 0,5 y 9/100.000 por año, es dos veces más frecuente en las mujeres y se presenta generalmente después de los 40 años ${ }^{1,2}$. El pronóstico del cáncer diferenciado de tiroides (CDT), excluida la variedad medular, es bueno, con una tasa de sobrevida a 10 años que excede el $90 \%{ }^{3}$. Según sus caracteres morfológicos se subdivide en cáncer papilar $(\mathrm{CP})$ y cáncer folicular $(\mathrm{CF})$, considerándose a este último mucho más agresivo. Dada la baja incidencia y larga sobrevida se requieren grandes poblaciones de pacientes, seguidas por muchos años (a veces más de 30), para identificar el efecto del tipo de tratamiento y los factores clínicos e histológicos asociados a un mal pronóstico.

Desde el punto de vista clínico, se considera factores de mal pronóstico para la sobrevida la edad mayor de 45 años al momento de la presentación de la enfermedad, el tamaño del tumor, la presencia de metástasis a distancia, y la resección incompleta del tumor ${ }^{4,5}$. Desde el punto de vista histológico se consideran factores de mal pronóstico la diferenciación del tumor (alta, media y baja), la permeación e invasión linfovascular ${ }^{4,6}$.

La prevalencia de metástasis a distancia a diez años de seguimiento varía entre $6 \%$ y $23 \%$ y, los sitios más frecuentes son pulmón y hueso ${ }^{7}$. Los objetivos del presente estudio son:

- Identificar y comparar la forma de presentación y evolución del CDT.

- Definir la curva de sobrevida actuarial a 10 años para el CP y el CF.
- Identificar los factores clínicos de mal pronóstico del CDT.

- Identificar los factores histológicos de mal pronóstico del CDT.

\section{Material y Método}

Tipo de estudio: Cohorte única longitudinal.

Criterios de inclusión:

a. Pacientes con el diagnóstico de cáncer diferenciado de tiroides, sin tratamiento previo, operados en el Servicio de Cirugía del Hospital Clínico San Pablo de Coquimbo y la Unidad de Oncología del Hospital San Juan de Dios de La Serena.

b. Pacientes que contaban con estudio y registro histopatológico completo en el Servicio de Anatomía Patológica del Hospital Clínico San Pablo de Coquimbo, unidad que procesa, registra y almacena toda la información de la especialidad de los hospitales de la red asistencial pública de la IV Región.

\section{Criterios de exclusión:}

a. Registros clínicos y/o histológicos extraviados o incompletos.

b. Casos detectados incidentalmente en autopsias.

c. Pacientes sometidos sólo a biopsias diagnósticas y/o que no fueron tratados con cirugía.

Período del estudio: Enero 1984-Diciembre 2007. La información relacionada con las operaciones, tratamientos complementarios y seguimiento fueron extraídas de las historias clínicas. Los datos histopatológicos fueron extraídos de los informes de 
biopsia. En el período se identificaron 105 pacientes. En 16 casos hubo registros clínicos extraviados o incompletos. En consecuencia este estudio incluyó 89 pacientes. En los casos fallecidos, su condición fue confirmada en el Registro Civil e Identificación.

Clasificación de los tumores: Se hizo según los criterios TNM de la OMS que consideran ${ }^{3}$ :

T: Tamaño del tumor

$\mathrm{T}_{\mathrm{x}}$ sin datos.

$\mathrm{T}_{0}^{\mathrm{x}}$ no palpable.

$\mathrm{T}_{1}<1 \mathrm{~cm}$.

$\mathrm{T}_{2} \quad 1-4 \mathrm{~cm}$.

$\mathrm{T}_{3}>4 \mathrm{~cm}$.

$\mathrm{T}_{4}$ cualquier tamaño con invasión extratiroídea.

$\mathrm{N}$ : Estado de linfonodos

$\mathrm{N}_{\mathrm{x}}$ sin información.

$\mathrm{N}_{0}^{\mathrm{x}}$ cervical no palpable.

$\mathrm{N}_{1}$ adenopatía cervical ipsilateral.

$\mathrm{N}_{2}$ adenopatía contralateral o bilateral.

$\mathrm{N}_{3}$ adenopatías cervicales fijas.

M: Metástasis

$\mathrm{M}_{\mathrm{x}} \sin$ información.

$\mathrm{M}_{0}^{\mathrm{x}} \sin$ metástasis a distancia.

$\mathrm{M}_{1}$ metástasis a distancias.

Estadio de la enfermedad:

Menor de 45 años

Estadio I

Estadio II

cualquier $\mathrm{T}$, cualquier $\mathrm{N}, \mathrm{M}_{0}$. cualquier $\mathrm{T}$, cualquier $\mathrm{N}, \mathrm{M}_{1}$.

Mayor de 45 años

Estadio I

Estadio II

Estadio III

Estadio IV

$$
\begin{aligned}
& \mathrm{T}_{1}, \mathrm{~N}_{0}, \mathrm{M}_{0^{\circ}} \\
& \mathrm{T}_{2}, \mathrm{~N}_{0}, \mathrm{M}_{0^{\circ}} \\
& \mathrm{T}_{3}, \mathrm{~N}_{0^{\prime}}, \mathrm{M}_{0^{\circ}} \\
& \mathrm{T}_{4}, \mathrm{~N}_{0}, \mathrm{M}_{0^{\circ}} \\
& \text { cualquier T, } \mathrm{N}_{1}, \mathrm{M}_{0^{\circ}} . \\
& \text { cualquier T y cualquier } \mathrm{N}, \mathrm{M}_{1} .
\end{aligned}
$$

Tratamiento quirúrgico: En presencia de un diagnóstico intraoperatorio de CDT el tratamiento fue tiroidectomía total. En presencia de linfonodos histológicamente comprometidos en el estudio de biopsia por congelación, se efectuó una disección linfática selectiva lateral de los grupos II, III y IV, conservando la vena yugular interna. En presencia de linfonodos clínicamente metastáticos se efectuó disección radical de cuello modificada, que incluyó los grupos de linfonodos II, III, IV y V, con conservación de nervio accesorio y músculo esternocleidomastoideo ${ }^{8,9}$. En aquellos pacientes con diagnóstico confirmatorio post operatorio de cáncer, se completó la tiroidectomía en un segundo tiempo.
Tratamiento complementario: Luego de la tiroidectomía se efectuó tratamiento con radio yodo (80$150 \mathrm{~m} \mathrm{Ci}$ de acuerdo al peso del paciente y extensión del tumor) y supresión-sustitución con tiroxina 100-150 microgramos/día (8), según los valores $\mathrm{T}_{4}$ y TSH sérico.

El seguimiento se hizo con tiroglobulina plasmática, ecografía de cuello, radiografía de tórax y cintigrafía de cuerpo completo. Los pacientes fueron controlados 3-4 veces al año por 5 años y luego 1 vez al año.

\section{Definiciones:}

a) recurrencia local y/o regional: enfermedad clínicamente detectable en el lecho del tiroides o linfonodos cervicales.

b) metástasis a distancia: enfermedad detectable clínica o radiológica en órganos distintos al lecho de la glándula o linfonodos cervicales.

Desenlace: Para cerrar el estudio es establecieron las siguientes opciones:

Vivo sin enfermedad, vivo con metástasis locales y regionales, vivo con metástasis a distancia, vivo con metástasis en linfonodos y a distancia, fallecido por la enfermedad, fallecido por otra causa.

Análisis estadístico: Los datos fueron recolectados en una base de datos EPI Info 3.5. Se utilizó análisis univariable para comparar los variables de los grupos con distinto desenlace. Todas las dócimas de significancia se obtuvieron con dos colas. Las variables continuas se compararon utilizando la $\mathrm{t}$ de Student para las variables distribuidas normalmente. Se utilizó la prueba estadística de chi cuadrado o la prueba exacta de Fischer para comparar variables categóricas. Se consideró significativo un valor de $\mathrm{p}=<0,05$.

\section{Resultados}

La presentación del cáncer papilar y folicular en los 89 pacientes (76 CP Y $13 \mathrm{CF}$ ) muestra una distribución similar en términos de edad, sexo, tamaño del tumor, presencia de linfonodos metastáticos y presencia de metástasis al momento del diagnóstico (Tabla 1). Al comparar 4 caracteres histológicos, identificamos características histológicas estadísticamente similares entre cáncer papilar y folicular (Tabla 2). En este grupo de pacientes no identificamos un signo clínico o histológico que orientara a reconocer el tipo histológico del cáncer. Se identificó un carcinoma mixto, papilar y folicular.

La mediana de seguimiento fue 59,3 meses (3 meses a 24 años). Un $75 \%$ de los pacientes tuvo 
Tabla 1. Demografía y presentación clínica. Cáncer diferenciado de tiroides

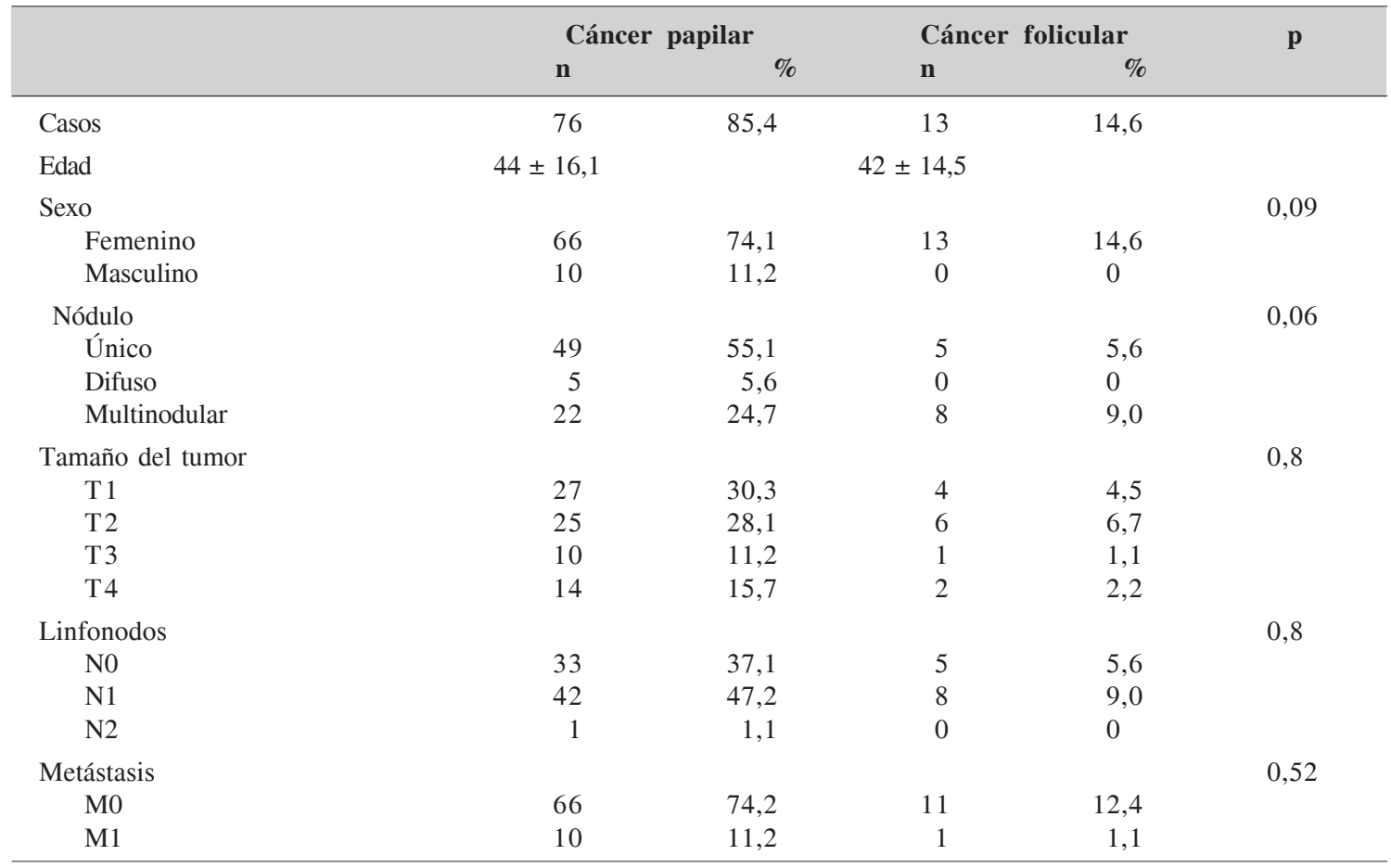

Tabla 2. Características histopatológicas. Cáncer diferenciado de tiroides

\begin{tabular}{|c|c|c|c|c|c|}
\hline & \multicolumn{2}{|c|}{ Cáncer papilar } & \multicolumn{2}{|c|}{ Cáncer folicular } & \multirow[t]{2}{*}{$\mathbf{p}$} \\
\hline & $\mathbf{n}$ & $\%$ & $\mathbf{n}$ & $\%$ & \\
\hline Casos & 76 & 85,4 & 13 & 14,6 & \\
\hline Extensión extracapsular & & & & & 0,39 \\
\hline No & 50 & 56,2 & 8 & 9,0 & \\
\hline Sí & 26 & 29,2 & 5 & 5,6 & \\
\hline Permeación linfovascular & & & & & 0,40 \\
\hline No & 44 & 49,4 & 7 & 7,9 & \\
\hline Sí & 32 & 35,9 & 6 & 6,7 & \\
\hline Infiltración perineural & & & & & 0,68 \\
\hline No & 70 & 18,7 & 12 & 13,5 & \\
\hline Sí & 6 & 6,7 & 1 & 1,1 & \\
\hline Multifocalidad & & & & & 0,41 \\
\hline No & 70 & 78,7 & 9 & 10,1 & \\
\hline Sí & 6 & 6,7 & 4 & 4,5 & \\
\hline
\end{tabular}

una mediana de seguimiento mínimo de 7 años. Al cierre del seguimiento el $86,5 \%$ de los pacientes $(65$ pacientes con $\mathrm{CP}$ y 12 con $\mathrm{CF}$ ) se encontraban vivos y libres de enfermedad; 5 casos con CP y 1 con $\mathrm{CF}$ presentaron recurrencia regional o a distancia. El sitio de recurrencia a distancia fue mediastino, pulmón y hueso (Tabla 3); el período medio de in- tervalo libre de enfermedad hasta la aparición de recurrencia fue 52,7 meses (rango: 3 meses-16 años). Siete pacientes fallecieron.

La sobrevida actuarial global de Kaplan y Meier a los 10 años para cáncer papilar, fue de $84 \%$ y, para cáncer folicular de $100 \%$. Al segregar el CP por estadios, encontramos a los 10 años de segui- 
Tabla 3. Desenlace de pacientes. Cáncer diferenciado de tiroides

\begin{tabular}{lrcr}
\hline & Papilar & Folicular & $\%$ \\
\hline Vivo sin enfermedad & 65 & 12 & 86,5 \\
Vivo con metástasis linfática & 1 & 0 & 1,1 \\
Metástasis a distancia & 3 & 1 & 4,5 \\
$\quad$ Mediastino & 1 & & \\
$\quad$ Pulmón* & 1 & 2 & \\
$\quad$ Hueso & & 1 & \\
Fallecido por la enfermedad & 6 & 0 & 6,7 \\
Fallecido por otra causa & 1 & 0 & 1,1 \\
Total & 76 & 13 & \\
\hline
\end{tabular}

* Metástasis presentes en más de un sitio.
Tabla 4. Factores de riesgo de mortalidad. Cáncer diferenciado de tiroides

\begin{tabular}{llll}
\hline Factores de riesgo & p & R.R. \\
\hline Tamaño de tumor & T2 & n.s & 1,1 \\
& T3 & n.s. & 1,52 \\
& T4 & 0,05 & 1,6 \\
Metástasis ganglionar & n.s. & 1,22 \\
Metástasis a distancia & 0,02 & 9,09 \\
Mayor de 45 años & n.s. & \\
Invasión extracapsular & n.s. & 1,13 \\
Invasión linfática y/o vascular & 0,02 & 2,65 \\
Multifocalidad & n.s. & 1,36 \\
\hline
\end{tabular}

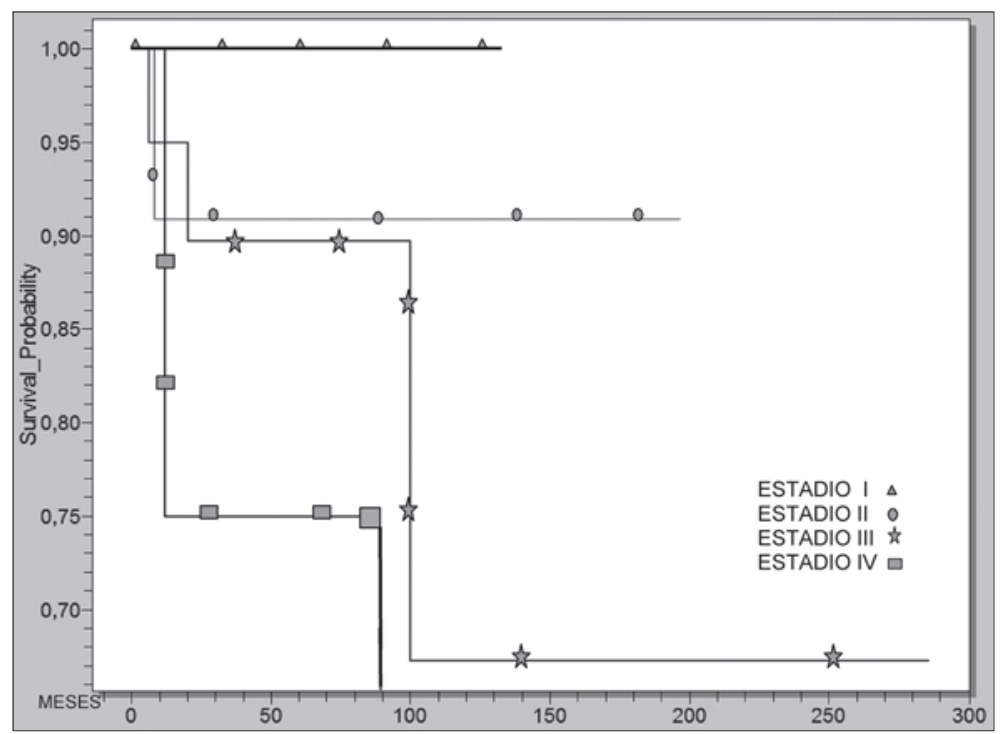

Figura 1. Sobrevivencia actuarial 10 años cáncer diferenciado de tiroides. Hospitales de Coquimbo - La Serena 1984-2007. miento una sobrevida de $100 \%$ en estadio I, $92 \%$ en estadio II, $68 \%$ en estadio III y $0 \%$ de sobrevida en estadio IV (Figura 1).

Dado que no hubo mortalidad en cáncer folicular, el análisis de signos clínicos e histológicos de mal pronóstico se restringe al grupo de pacientes con cáncer papilar. La mortalidad se asocia significativamente a la presencia de metástasis $(\mathrm{p}=0,02)$, tumores T4 $(\mathrm{p}=0,05)$ e infiltración neoplásica vascular y linfática $(\mathrm{p}=0,02)$. Otros factores como compromiso de linfonodos y caracteres histológicos como invasión extracapsular o multifocalidad no mostraron diferencia entre sobrevivientes y fallecidos. Sin embargo, el RR de mortalidad por metástasis en linfonodos es de 1,2 y con metástasis a dis- tancia es de 9,1. La presencia de T4 es 1,6 y para la presencia de invasión tumoral linfática y vascular genera RR 2,6. Para la multifocalidad al interior del tiroides produce un RR 1,4 (Tabla 4).

En 25 pacientes identificamos complicaciones del tratamiento quirúrgico: hipoparatiroidismo definitivo 15 casos $(16,8 \%)$ con suplemento de calcio oral entre 1 y 3 gramos diarios; parálisis del nervio laríngeo recurrente en 7 casos $(7,8 \%)$; hematoma de la herida y seroma 2 casos $(2,2 \%)$, infección de herida 1 caso $(1,1 \%)$ y sin complicaciones 60 casos $(68,5 \%)$. Un $65 \%$ de las complicaciones definitivas $(15 / 23$ casos) ocurrieron entre 1984 y 1993. Desde 1998 hubo 1 caso de lesión de nervio laríngeo recurrente y un caso de hiopoparatiroidismo. 


\section{Discusión}

Desde el año 2000 hasta la fecha hay pocas publicaciones chilenas referidas a la evolución, pronóstico y desenlace del cáncer diferenciado de tiroides ${ }^{8-10}$, hecho explicable por la baja frecuencia de la enfermedad y la consiguiente dificultad para reunir una serie de pacientes. Este hecho nos ha llevado a presentar la experiencia conjunta de dos hospitales que actúan en forma complementaria en la red asistencial pública de la IV Región de Chile.

En esta serie encontramos una relación $\mathrm{CP} / \mathrm{CF}$ de 5:1 y un predominio de las mujeres sobre los varones de 5:1, proporción para el $\mathrm{CDT}^{1,2}$ más alta que la descrita convencionalmente, de 3:2, resultado probable de una muestra pequeña. $\mathrm{Al}$ analizar la edad y las características T, N, y M de la enfermedad, nuestros datos no identificaron un atributo clínico que permita hacer un diagnóstico diferencial entre cáncer papilar y folicular antes del análisis de la biopsia. Estas características demográficas son similares a las de otras publicaciones nacionales $^{9,11}$. Tampoco identificamos un atributo histológico específico para reconocer cada presentación del CDT.

El 86,5\% de los pacientes vivos al cierre del seguimiento está dentro de lo descrito en publicaciones extranjeras ${ }^{2,3}$ y la literatura chilena ${ }^{8,9}$, que señala sobrevida entre 60 y $95 \%$. La curva de sobrevida a 10 años de nuestra serie de pacientes portadores de cáncer papilar es similar a las descritas por otros autores $^{2,4,6,9-11}$, destacando una sobrevida de $100 \%$ para la etapa I y $0 \%$ para los cánceres avanzados estadio IV. Este último grupo de pacientes tiene una expectativa máxima de vida de 6 años. Nuestra serie muestra un mejor pronóstico en el grupo de CF (sobrevivencia de 100\%), en oposición a lo señalado por diversos autores ${ }^{2,3,6,8,10}$, lo que se explica por dos razones: porque la mayoría de estos pacientes correspondía a estadios tempranos de la enfermedad ( $<45$ años y tumores T1 y T2) y, porque son pocos pacientes (13 casos). Los signos histológicos de mal pronóstico de nuestra serie se ajustaron a lo conocido ${ }^{4,6,8}$.

\section{Conclusiones}

Aunque el seguimiento de esta casuística es algo reducido, los resultados sugieren que el riesgo de muerte se asocia, fundamentalmente, a compromiso histológico linfático y metástasis a distancia y, en menor grado, al avance local y compromiso de linfonodos cervicales; por otra parte, el planteamiento terapéutico quirúrgico ha resultado efectivo para el control de la enfermedad local y para el control de las metástasis linfáticas regionales del $\mathrm{CP}$, que tiene una conocida diseminación por esta vía.

La incidencia de complicaciones post operatorias dobla las descritas por los autores nacionales ${ }^{8-11}$. La ocurrencia de dos tercios de las complicaciones definitivas en el primer tercio de la serie refleja la curva de aprendizaje del equipo de cirujanos hasta alcanzar un rendimiento óptimo. Desde el año 1998 el tratamiento quirúrgico del CDT en ambos hospitales se ha concentrado en cuatro especialistas con una frecuencia de mínimas complicaciones (OI, NV, $\mathrm{PH}, \mathrm{MV})$.

\section{Referencias}

1. Jameson L, Weetman A. Trastornos de la glándula tiroides. En Harrison: Principios de Medicina Interna 2006; $16^{\text {a }}$ Edición. MacGraw Hill, Vol 2: 2314-2340.

2. Chow Sin-Ming, Law S, Au Siu Kie, Leung To-Wai, Chan P. Mendenhall W. Differentiated thyroid carcinoma. Comparison between papillary and follicular carcinoma in a single institute. Head \& Neck 2002; 24: 670-677.

3. Lundgren CI, Hall P, Dickman PW, Zedenius J. Clinically significant prognostic factors for differenciated thyroid carcinoma. Cancer 2006; 106: 524531.

4. Lo Chung Yau, Chan Wai-Fan, Lam Kim-Yin, Wan Koon-Yat. Follicular thyroid Carcinoma: the role of histology and staging systems in predicting survival. Ann Surg 2005; 242: 708-715.

5. Gemsenjäger E, Heitz $P$, Steifert $P$, Martina B, Schweizer I. Differentiated thyroid carcinoma. Swiss Med Wkly 2001; 131: 157-163.

6. Lerch H, Schober O, Kuwert T, Saur HB. Survival of differentiated thyroid carcinoma studied in 500 patients. J Clin Oncol 1997; 15: 2067-2075.

7. Benbassat C, Mechlis-Frish S, Hirsch D. Clinicopathologycal characteristics and long term outcome in patients with distant metastases from differentiated thyroid cancer. World J Surg 2006; 30: 1088-1095.

8. Torres J. Cancer diferenciado de tiroides. Rev Chil Cir 2000; 52: 211-218.

9. Pérez JA, Poblete MT, Ramírez R, Henning E, Uherek F, Salem C, y col. Carcinoma diferenciado de tiroides. Rev Chil Cir 2000; 52: 585-592.

10. González M, Andrades P, Contreras O, Irarrázabal V, Paladines Z, Inturias R, y col. Sobrevida en cáncer de tiroides: seguimiento de 365 pacientes por un período de 37 años. Rev Chil Cir 2002; 54: 329-335.

11. Piraíno P, Sepúlveda A, Lillo R, Pineda P, Liberman C. Cáncer tiroídeo. Comunicación de 85 casos. Rev Méd Chile 2000; 128: 405-410. 